Liverpool Preprint: LTH 349

hep-lat/9506009

5 June 1995

\title{
Is the Quenched Spectrum in agreement with Experiment?
}

\author{
UKQCD Collaboration \\ P. Lacock and C. Michael \\ DAMTP, University of Liverpool, Liverpool, L69 3BX, U.K.
}

\begin{abstract}
We analyse the meson spectrum in quenched QCD using lattice gauge theory. By studying hadron propagation with a variety of operators (both smeared and local), we are able to extract the ground state and first excited state masses with confidence. We pay attention to the correlations among the data used in the fits to extract these masses and couplings. We compare the resulting hadron spectrum with experiment and find evidence for a significant departure in the pseudoscalar and vector meson masses.
\end{abstract}

\section{Introduction}

Lattice gauge theory is able to evaluate the hadron spectrum from first principles. In practice, the quenched approximation is needed to keep the computational resource manageable. Thus it is valuable to calibrate the quenched approximation. Since data from different fermionic actions and different lattice spacings need to be combined, it is worthwhile to look for observables which are independent. This is the motivation for the Edinburgh plot of $m_{N} / m_{\rho}$ versus $m_{\pi} / m_{\rho}$ for example. Results for the baryon spectrum are less precise than those for mesons, partly because the baryonic states are heavier. Thus it will be helpful to construct combinations of mesonic masses (pseudoscalar $\mathrm{P}$ and vector $\mathrm{V}$ in particular) to compare with experiment. Typically, $m_{\rho}$ is used to set the lattice spacing scale $a$ and $m_{V} / m_{P}$ is used to fix the quark mass. The only remaining information is on the relative quark mass dependence of the vector and pseudoscalar mesons. We thus focus on this and propose to use

$$
J=m_{K^{*}} \frac{d m_{V}}{d m_{P}^{2}}
$$


as a measure. This is in the spirit of the Maiani-Martinelli proposal [1, 2] to use $\left(m_{K^{*}}-\right.$ $\left.m_{\rho}\right) /\left(m_{K}^{2}-m_{\pi}^{2}\right)$ as an independent way to determine the lattice scale. We also follow the suggestion of the GF11 group [3] to avoid the errors inherent in a chiral extrapolation by making the comparison at the quark mass which reproduces the mass of the $\mathrm{K}$ and $\mathrm{K}^{*}$ mesons. As well as the vector and scalar mesons, results for the $J^{P C}=1^{++}$axial mesons are also compared to experiment.

We present our approach in the context of the quark model in section 2. The new lattice results for the meson masses are then discussed in section 3. After a comparison with other lattice determinations in section 4 , we present our conclusions.

\section{The quark model}

We shall study hadronic states using lattice techniques for a range of quark masses. Thus we need to establish mass formulae for the dependence of the mass of a meson on the masses $\left(q_{1}\right.$ and $q_{2}$ ) of the quarks of which it is composed.

The simplest assumption is that the meson masses are given by a Taylor series in these quark masses. Assuming that the linear approximation is sufficient, for the vector mesons, this implies that

$$
m_{V}\left(q_{1}, q_{2}\right)=d+c\left(q_{1}+q_{2}\right)
$$

Here the coefficient of $q_{1}$ and of $q_{2}$ is the same from symmetry.

For the pseudoscalar mesons, chiral symmetry considerations imply that the appropriate expression is

$$
m_{P}^{2}\left(q_{1}, q_{2}\right)=b\left(q_{1}+q_{2}\right)
$$

We shall be concerned here with light quarks: $u$, d and s. To avoid consideration of the small isospin violating effects, we use the isospin averaged mass values and treat the $\mathrm{u}$ and $\mathrm{d}$ quarks as having the same mass (non-strange: ns). Then to have a check of these mass formulae, one needs to consider the mixing of the experimental states with isospin 0. Taking the usual assumption that the $\phi$ meson is purely composed of $\mathrm{s}$ quarks while the $\omega$ meson has purely non-strange quarks (and so is degenerate in mass with the $\rho$ meson), these relations yield an equal mass splitting between $\rho, \mathrm{K}^{*}$ and $\phi$ mesons. This is in close agreement with data since the mass differences of $\phi-\mathrm{K}^{*}$ and $\mathrm{K}^{*}-\rho$ are equal within $4 \%$. Note, however, that the differences $\mathrm{K}^{*}-\rho$ and $\mathrm{K}^{*}-\omega$ differ by $10 \%$ which shows that the assumption of ideal mixing of the $\phi$ and $\omega$ is not perfect.

Further checks of these mass formulae can be made from lattice studies where the quark masses can be adjusted freely. The most accurate lattice spectroscopy is currently available in the quenched approximation. Although the chiral behaviour in the quenched approximation will 
be modified [4, [], this effect is expected to be significant only at very small quark masses. Thus it is appropriate to compare the above mass formulae with quenched lattice mass values without extrapolating to the chiral limit. In order to set a common reference quark mass we shall choose

$$
m_{V}=1.8 m_{P}
$$

which corresponds to the experimental $\mathrm{K}^{*} / \mathrm{K}$ mass ratio. We define the values of the vector and pseudoscalar masses at this quark mass as $m_{v}$ and $m_{p}$ respectively.

Equations 1 and 2 imply that a plot of $m_{V}$ against $m_{P}^{2}$ will be a straight line for varying quark masses. This has been checked in lattice studies where a range of values of $q_{1}$ and $q_{2}$ are used. Moreover, studies with $q_{1} \neq q_{2}$ have also been conducted [2, 6] and they show excellent agreement with the mass formulae above. A compilation of data at $\beta=6.0$ for Wilson fermions is shown in fig. 1 and at $\beta=6.2$ for the clover fermionic action in fig. 2 .

In lattice studies, it is usual to relate the quark mass to bare lattice parameters (eg. it is assumed to be linear in $1 / K$ for Wilson fermions where $K$ is the Wilson hopping parameter). Furthermore the scale of masses involves the lattice spacing $a$. Thus the pseudoscalar and vector meson masses are commonly used to fix the relationship of $K$ to the quark mass and to fix $a$. This fixing procedure tends to obscure the underlying agreement between the experimental spectrum and the lattice spectrum. Here we wish to emphasize that there is one combination of the parameters in the above mass formulae which is independent of $a$ and of $K$ and which does not involve extrapolation to the chiral limit. This is the dimensionless combination

$$
J=m_{v} \frac{c}{b}=m_{v} \frac{d m_{V}}{d m_{P}^{2}}
$$

Thus, as was emphasized by the GF11 group [3], one can compare lattice results with the experimental spectrum without having to extrapolate to the chiral limit. The combination $J$ can be determined in principle by lattice results at two quark masses. Thus one parametrises the variation of $m_{V}$ with $m_{P}^{2}$ as a straight line. Then the intersection of this line with $m_{V}=1.8 m_{P}$ determines the reference value $m_{v}$ which is to be multiplied by the slope to yield $J$. The useful feature of $J$ is that it is independent of the quark mass values chosen to determine it, provided that the relationship of $m_{V}$ to $m_{P}^{2}$ is linear for those quark masses.

We can use the experimental spectrum of $\pi, \mathrm{K}, \rho, \omega, \mathrm{K}^{*}$ and $\phi$ mesons with masses 138.0, 495.7, 770, 781.9, 892.1, $1019.5 \mathrm{MeV}$ respectively to determine the coefficients in the mass formulae. These values are consistent with the mass formulae, treating the mesons as made from two types of quark: strange $q_{s}$ and non-strange $q_{n s}$. One finds $q_{n s} / q_{s}=0.04$. We use the $\mathrm{K}^{*}, \rho$ and $\mathrm{K}, \pi$ differences to determine $d m_{V} / d m_{P}^{2}$ since this does not depend on mixing with the isoscalar states. This gives the dimensionless combination

$$
J=0.48(2) \text {. }
$$


The error on this has been estimated from the $\phi-\mathrm{K}^{*}$ to $\mathrm{K}^{*}-\rho$ mass ratio which is 1.04 .

We shall also study the $1^{++}$mesons. The observed states are the $\mathrm{a}_{1}$ meson of mass $1230(40)$ $\mathrm{MeV}$ and the $\mathrm{f}_{1}$ mesons of mass $1285(5)$ and $1427(2) \mathrm{MeV}$. If we assume that the heavier $\mathrm{f}_{1}$ meson is predominantly $s \bar{s}$, then the strange partner $\mathrm{K}_{A}^{*}$ would have mass $1328(20) \mathrm{MeV}$. The experimental situation regarding the strange $1^{+}$mesons is confused by the mixing between the mesons from the $1^{++}$and $1^{+-}$nonets to give two observed states with masses $1273(7)$ and 1402(7) $\mathrm{MeV}$. In the absence of a predictive model for this mixing, we take the above value of $1328(20) \mathrm{MeV}$ as the mass of the $\mathrm{K}_{A}^{*}$. Thus at $m_{V}=1.8 m_{P}$, experiment gives the ratio $m\left(\mathrm{~K}_{A}^{*}\right) / m\left(\mathrm{~K}_{V}^{*}\right)=1.48(2)$.

We now discuss quenched lattice determinations of the meson spectrum.

\section{Lattice Measurements}

The results we shall present come from a study of light quark propagators in the 60 configurations of size $24^{3} \times 48$ at $\beta=6.2$ obtained by UKQCD [2]. These are at hopping parameters $K=$ 0.14144 and $K=0.14226$ using the clover improved fermion action. The new feature of the present analysis is that the quark propagators were determined with smeared sources and sinks. The Jacobi smearing algorithm was used [7] with $K_{S}=0.190$ and $N=90$. This is a gauge invariant smearing prescription. The notation we shall use is that SL corresponds to (all) quarks smeared at the source but local at the sink, etc. We measured SS and SL correlations and previous results [2] for the LL correlations were also used in the analysis.

For the mesonic operators at source and sink, we used several spin combinations. For the pseudoscalar mesons, both the usual pseudoscalar operator (P) $\bar{q} \gamma_{5} q$ and the axial operator (A) $\bar{q} \gamma_{5} \gamma_{4} q$ were used. Moreover we employed both $\mathrm{P}$ and $\mathrm{A}$ operators at both source and sink, so yielding 4 combinations PP, AA, PA and AP. Since this was carried out for the three smearing choices, this yielded 12 different quantities which could be used to explore the pseudoscalar meson spectrum and couplings. For the vector meson channel we found that extra spin combinations (i.e. using $\gamma_{i} \gamma_{4}$ as well as the usual $\gamma_{i}$ ) did not help since they had very large statistical errors. For the axial vector meson channel, we used the axial current as an operator and analysed results with only a smeared source but local and smeared sink.

The advantage of having many operators available is that it makes the fit to extract the mass values much more tightly constrained. This can be seen since the euclidean time formulation implies that eigenstates of the transfer matrix contribute to hadronic correlators $\left\langle H^{a}(0) H^{b}(t)>\right.$ as

$$
\begin{aligned}
C_{a b}(t) & =<H^{a}(0) H^{b}(t)> \\
& =h_{0}^{a} h_{0}^{b}\left(e^{-m_{0} t}+e^{-m_{0}(L-t)}\right)+h_{1}^{a} h_{1}^{b}\left(e^{-m_{1} t}+e^{-m_{1}(L-t)}\right)+\ldots
\end{aligned}
$$


where $h_{i}^{c}$ is the amplitude to produce eigenstate $i$ from operator $H^{c}$. The expression is written assuming periodic boundary conditions in time for bosonic eigenstates - the AP and PA spin combinations for pseudoscalar mesons will need anti-periodic time boundary conditions.

Because of the factorisation of the coefficients above, if $D$ different operators are used at source and sink (so that $a, b=1, \ldots, D$ above) and $T$ different $t$-values are fitted, there will be $\frac{1}{2} D(D+1) T$ observables to fit with $(D+1) M$ parameters, where $i=1, \ldots, M$ eigenvalues are retained. Thus the number of observables increases faster than the number of parameters as $D$ increases. This makes it feasible to retain more eigenvalues, which, in turn, allows a larger $t$-region to be fitted. Furthermore, the fitted $t$-region extends to smaller $t$-values where the relative errors are smaller.

This conclusion that several observables should be studied simultaneously can also be motivated by noting that the ground state is only determined accurately when an estimate of the first excited state is available. This is necessary since the energy difference controls the rate of approach of $C_{a b}(t)$ to the expression given by the ground state component alone. However, fitting 2 (or more) exponentials to just one function $C(t)$ is not very stable: better is to have several such functions (provided that they do indeed have different relative amounts of ground state and excited state).

The advantage of measuring several independent observables has long been known in the lattice gauge theory community. It is the root of the success of the variational method and is de rigeur for studies of glueballs and potentials. The variational method has also been used to study the light quark spectrum [7]. Because the data for different observables and for different $t$-separations are very highly correlated statistically, we prefer to use a general correlated fitting program in the present work. The advantages of improved determination of the spectrum are still retained.

The correlations among the hadronic Green functions $C_{a b}(t)$ are significant, particularly for adjacent values of $t$. As discussed in ref [\&], this has important consequences for estimating the goodness of fit. Since we fit, for the pseudoscalar channel, over 200 data points simultaneously, we cannot use a full correlated $\chi^{2}$ approach since there are only 60 independent configurations available. Methods for accommodating the essence of the correlation among the data, while using less parameters, have been proposed [8]. Here we use the method of the 5-diagonal approximation to the inverse correlation matrix as our basic tool. This method [8] copes with the strong correlations in $t$ very economically - it uses only two parameters for each type of observable. To check on possible systematic errors, we use two more extreme assumptions as a guide. These are a completely uncorrelated fit and a fit allowing correlations among the data in both $t$ and between different observables (ie $a$ and $b$ in our notation). This latter approach used a smoothing of the full correlation matrix by retaining the 12 largest eigenmodes and replacing the eigenvalues of the remaining modes by their average value - see ref [ 8$]$.

Errors were obtained by taking bootstrap samples of configurations from the original 60 . 
For quantities involving fits from different quark masses ( $K$-values) or for different quantum numbers ( $\mathrm{P}, \mathrm{V}$ or $\mathrm{A})$, we used the same set of bootstrap configurations so that a bootstrap error analysis of the final quantity of interest was obtained.

For each meson, we used a two-state fit. As discussed above, one state fits only are acceptable for a limited range of $t$ (i.e. larger $t$ ), while we found three state fits to be unstable. The $t$-range was chosen by requiring the $\chi^{2}$ per degree of freedom of our basic fit to be acceptable (i.e. close to 1.0). Because the evaluation of the goodness of fit can be difficult with highly correlated data, we are aware that the appropriate $t$-range might be somewhat wider or narrower. Since including smaller $t$-values tends to increase the fitted mass values, we took account of this source of systematic errors by varying the $t$-range fitted.

The results for the two state fits to the pseudoscalar and vector mesons are presented in Table 1 and shown in figures 3 to 7 . These results are compatible within the quoted errors with the earlier analysis [2] using local operators and making one state fits (i.e. plateau fits in the effective mass) to much smaller $t$-ranges. For the axial mesons, we found that a two state fit was not very stable, so we fixed the mass of the excited state at $1 \mathrm{GeV}$. The results are shown in Table 1.

\begin{tabular}{|c|c|c|c|c|c|}
\hline$K$ & $t$-range & $m_{P}$ & $m_{P}^{\prime}$ & $f_{P} / Z_{A}$ & $\chi^{2} /$ dof \\
\hline 0.14144 & $7-23$ & $0.297(1.7)$ & $0.679(41)$ & $0.0620(14)$ & $195 / 194$ \\
mixed & $7-23$ & $0.258(2.6)$ & $0.628(63)$ & $0.0566(15)$ & $183 / 194$ \\
0.14226 & $7-23$ & $0.212(5)$ & $0.530(104)$ & $0.0508(24)$ & $181 / 194$ \\
"K" & & $0.182(6)$ & & $0.0476(20)$ & \\
\hline$K$ & $t$-range & $m_{V}$ & $m_{V}^{\prime}$ & $1 /\left(f_{V} Z_{V}\right)$ & $\chi^{2} /$ dof \\
\hline 0.14144 & $7-23$ & $0.389(4)$ & $0.835(45)$ & $0.311(6)$ & $42 / 45$ \\
mixed & $7-23$ & $0.365(5)$ & $0.815(49)$ & $0.322(7)$ & $34 / 45$ \\
0.14226 & $7-23$ & $0.340(7)$ & $0.796(55)$ & $0.336(9)$ & $27 / 45$ \\
"K"" & & $0.327(11)$ & & $0.343(9)$ & \\
\hline$K$ & $t$-range & $m_{A}$ & $m_{A}^{\prime}$ & $1 /\left(f_{A} Z_{A}\right)$ & $\chi^{2} /$ dof \\
\hline 0.14144 & $4-15$ & $0.569(10)$ & 1.0 & $0.178(19)$ & $15 / 19$ \\
0.14226 & $4-15$ & $0.534(15)$ & 1.0 & $0.195(20)$ & $15 / 19$ \\
"K ${ }_{A}^{*}$ " & & $0.524(17)$ & & $0.200(21)$ & \\
\hline
\end{tabular}

Table 1: The pseudoscalar, vector and axial vector meson masses and couplings in lattice units ("mixed" refers to mesons with one quark of each hopping parameter $K$ ). The rows marked $\mathrm{K}$, $\mathrm{K}^{*}$ and $\mathrm{K}_{A}^{*}$ refer to an extrapolation to a quark mass such that $m_{V}=1.8 m_{P}$.

From these values we can calculate the dimensionless combination $J=0.37(3)$ where the error here is statistical. The main source of error in the determination of $J$ comes from the difference of vector meson masses $\left(a m_{V}\left(q_{1}\right)-a m_{V}\left(q_{2}\right)\right)$. The systematic error on this vector meson mass difference of 0.037 from using different $t$-ranges and different correlated fitting procedures is \pm 0.002 which is $5 \%$. Thus one can express the error on $J$ by adding a second 
systematic error as $J=0.37(3)(2)$. As can be seen from fig. 2, the UKQCD data on $m_{V}$ versus $m_{P}^{2}$ is consistent with a straight line but also allows some small curvature. This introduces a further systematic error since the slope and intercept are required at $m_{V} / m_{P}=1.8$ which is an extrapolation from the three smeared-source data points used above. This source of systematic error is hard to quantify since statistically significant evidence for any curvature does not exist. We suggest that $J=0.37(3)(4)$ is an appropriate final estimate from our quenched lattice study.

This value is significantly different from the experimental value of $J=0.48(2)$ given above. Since $J$ does not depend on the lattice spacing value used, nor on the critical hopping parameter $K_{c r i t}$, the discrepancy points towards an underlying difference between the quenched lattice and the full QCD experimental values.

There are also published data for the clover fermionic action at $\beta=6.2$ from the APE collaboration [9] using a $18^{3} \times 64$ lattice. Their results for $a m_{V}$ and $a m_{P}$ versus $K^{-1}$ disagree with those published by UKQCD [2] and confirmed above. The APE results were obtained from a one state fit (plateau fit) to the local correlators (LL in our notation). Such a fit can be influenced by inadequate statistics since the signal is relatively noisy at the large $t$-values (15 to 28) where the plateau in the effective mass is determined - see, for example the discussion in ref [10].

The disagreement in meson mass values is more pronounced at lower quark masses where the APE results for am are lower than the interpolated UKQCD values - see fig. 2. This might be caused by enhanced finite size effects (at spatial size $18^{3}$ compared to $24^{3}$ ) at such light masses. Such a conclusion is, however, in conflict with the results for staggered fermions [11] and Wilson fermions [3] that the am values are increased on a smaller spatial volume. A resolution of this disagreement would need higher statistics and/or the measurement of further correlations (such as smeared correlations) at the smaller volume.

Returning now to the axial vector meson spectrum, our results give $m_{A} / m_{V}=1.60(7)$ at a quark mass corresponding to $m_{V}=1.8 m_{P}$. This can be compared with the value of the ratio deduced from experimental data: 1.48(2) as discussed above. Again there is evidence for a discrepancy between the quenched lattice result and experiment. Since there has been relatively little study of this ratio in lattice work, the finite size and finite $a$ errors are not easy to estimate. Further work is again needed.

\section{Discussion}

Even though $J$ does not depend on the lattice spacing explicitly (it can be thought of as a mass ratio), it will have discretisation errors. These will be of order $a$ for the Wilson fermionic action and of order $\alpha_{S} a$ for the clover fermionic action. The other lattice approximation that needs to be checked is the finite lattice size used. 
Within the UKQCD collaboration we intend to study the discretisation and finite-size errors systematically. At present, we have data at one lattice size $\left(24^{3} \times 48\right)$ and at one $\beta$-value $(6.2)$ only. We chose these lattice specifications with the expectation that the discretisation and finite size errors would be very small for the light meson spectrum. To explore further the possible discretisation and finite size errors, we make some comparisons with other lattice determinations of the meson spectrum. To make a comparison using the clover fermionic action, we analyse the APE data [12] at $\beta=6.0$. Making a fit to their published results for $m_{V}$ versus $m_{P}^{2}$ for three light $K$-values, we obtain $J=0.37(2)$. We do not have access to the correlations among their results at different $K$-values, so the error estimate is based on an uncorrelated fit with the quoted diagonal errors only. Their lattice spatial size $\left(18^{3}\right)$ is comparable in physical volume to our work at $\beta=6.2$. Thus we can conclude that we see no sign of any $a$ dependence in $J$ as determined from quenched clover lattices. This is confirmation of our assumption above that the clover action should remove most of the $a$ dependence for $\beta>6.0$.

There are more extensive lattice data available for Wilson fermions. In this case, one would expect somewhat larger $a$ dependence to remain than in the clover case just discussed. We consider the extraction of $J$ from this data. One of the most comprehensive studies of the light meson spectrum has been made by the GF11 group [3]. Their largest $\beta$-value is 6.17 with lattice size $32^{2} \times 30 \times 40$. From their published results for $\left(m_{p}^{2} / m_{v}\right) d m_{V} / d m_{P}^{2}$, one can evaluate $J=0.373(13)$. This result is similar to the clover result for the nearby $\beta$-value of 6.2 . The GF11 group has also studied a sequence of lattices with coarser lattice spacing but similar physical size. The results for $J$ are shown in fig 8 by squares. The errors are sufficiently large that the results are compatible either with essentially no discretisation error ( $J$ independent of $a$ ) or with a substantial linear $a$ dependence which allows the experimental value to be obtained as the $a \rightarrow 0$ limit.

The GF11 data at $\beta=5.7$ have two different lattice spatial volumes. This enables the finite size effects to be explored. Again the data are consistent with no volume dependence of $J$ but have sufficiently large errors that they also would allow quite large volume dependence in principle. Using data at as low a $\beta$ value as 5.7 to determine the order $a$ finite size effects from Wilson fermions is also rather uncontrolled since the order $a^{2}$ discretisation effects from the pure gauge sector are known to be significant (over $30 \%$ in glueball to string tension ratio [14]) at 5.7 .

A study of finite-size effects can also be made by comparing the data at $\beta=6.0$ with Wilson fermions from the LANL group [6], QCDPAX group [13] and APE group [12]. The plot of $m_{V}$ against $m_{P}^{2}$ from these data is shown in fig. 1 . The spectra with different spatial lattice volumes $\left(32^{3}, 24^{3}\right.$ and $18^{3}$ respectively) are seen to be in agreement which implies that $J$ is independent of lattice spatial volume. The most comprehensive study is by the LANL group and fitting their data for $m_{V}$ versus $m_{P}^{2}$ yields $J=0.38(1)$. This result also confirms that there is no substantial finite size effect in the determination of $J$ since it agrees well with the GF11 data from smaller physical volumes at $\beta=6.17$ and 5.93 . 
Since the clover fermionic action is expected to have smaller discretisation errors than the Wilson action, the fact that the clover and Wilson discretisation results for $J$ agree for $\beta \geq 6.0$, suggests that there can be no substantial $a$ dependence in either. Furthermore all results, except the smaller volume GF11 determination at $\beta=5.7$, agree with $J=0.37$. Further precision data are needed to reinforce this conclusion that $J=0.37$ for quenched QCD.

To measure $J$ directly in lattice simulation with dynamical fermions will be hard. The usual procedure for dynamical fermion studies is to use sea quarks of one mass in such work. Then only $\operatorname{am}_{V}\left(q_{1}, q_{1}\right)$ and $\operatorname{am}_{P}\left(q_{1}, q_{1}\right)$ would be available. Making a direct comparison of meson masses at different dynamical quark mass values is inappropriate since the lattice spacing $a$ will depend on the sea-quark mass. A way forward is to use a partly quenched approach with a fixed seaquark mass but several valence quark masses. Typical results [15] use two flavours of staggered fermions as sea-quarks and Wilson valence quarks. The analysis of those data $\left(16^{3} \times 32\right.$ lattice at $\beta=5.6$ with $a m_{q}=0.01$ and 0.025$)$ yields $J=0.356(11)$ and $J=0.345(7)$ respectively from fitting the published mass values for $m_{V}$ versus $m_{P}^{2}$. These values are similar to those obtained above from quenched lattices. Indeed the authors point out that their results for light hadrons are indistinguishable from those in the quenched approximation. Thus it appears that the full QCD result will only be approached when the sea-quark masses used in the lattice studies are reduced further.

To explore dynamical quark simulation fully will need much more work. Implementing two different sea-quark masses will be complicated. Using realistic masses will be even harder. There are also indications of enhanced finite-size effects in dynamical quark studies.

\section{Conclusions}

Quenched QCD is an excellent model for the hadronic interactions. It is an approximation and the accuracy of the approximation can be estimated in some cases. For instance, the beta-function can be evaluated perturbatively and the quenched approximation amounts to treating $\left(33-2 N_{f}\right)$ as 33 , an error of $20 \%$. The width of the $\rho$ meson is zero in the quenched approximation, in contrast to the non-zero value in full QCD. Previous lattice studies in quenched QCD have claimed agreement [3] with the experimental light hadron spectrum. In this work, we have concentrated on a quantity $J$ which can be determined from the pseudoscalar and vector meson masses and which is independent of the lattice spacing and hopping parameters. We find evidence that $J=0.37(2)(4)$ from quenched lattice studies. This is in contrast to the experimental value of $J=0.48(2)$. Further lattice evaluations are needed to confirm the assumptions we have made about the discretisation and finite size errors on $J$. We also have presented some evidence for a discrepancy between the ratio of axial and vector meson masses from quenched lattices compared to experiment.

These discrepancies should be no cause for surprise. What it does show is that a careful study 
of lattice QCD with dynamical quarks is of importance if an accurate description is needed.

\section{Acknowledgements}

This research was supported by the UK Science and Engineering Research Council under grants GR/G 32779, GR/H 49191, GR/J 21200 and GR/H 53624, by the University of Edinburgh and by Meiko Limited. We are grateful to Edinburgh University Computing Service and, in particular, to Mike Brown for maintaining service on the Meiko i860 Computing Surface. We thank our colleagues in the UKQCD collaboration at Edinburgh for their assistance in making the data sets available to us. We acknowledge helpful advice from Chris Sachrajda and Hartmut Wittig. 


\section{References}

[1] L. Maiani and G. Martinelli, Phys. Lett. 178B (1986) 265.

[2] UKQCD Collaboration, C. R. Allton et al., Phys Rev D 49 (1994) 474.

[3] F. Butler et al., Nucl. Phys. B421 (1994) 217; B430 (1994) 179.

[4] S. Sharpe, Nucl. Phys. B (Proc. Suppl.) 17 (1990) 146.

[5] C. Bernard and M. Golterman, Nucl. Phys. B (Proc. Suppl.) 26 (1992) 360; Phys. Rev. D4 (1992) 853.

[6] T. Bhattacharya and R. Gupta, hep-lat/9501016, Nucl. Phys. B (Proc. Suppl.) (in press).

[7] UKQCD Collaboration, C. R. Allton et al., Phys. Rev. D47 (1993) 5128.

[8] C. Michael and A. McKerrell, Liverpool preprint LTH342, hep-lat/9412087, Phys. Rev. D (in press).

[9] C. R. Allton et al., Phys. Lett. B326 (1994) 295.

[10] C. Michael, Nucl. Phys. B (Proc. Suppl.)42 (1995) 147.

[11] S. Aoki et al., Nucl. Phys. B (Proc. Suppl.) 34 (1994) 363.

[12] C. R. Allton et al., Nucl. Phys. B413 (1994) 461.

[13] Y. Iwasaki et al., Nucl. Phys. B(Proc. Suppl) 34 (1994) 354.

[14] UKQCD Collaboration, G. Bali et al., Phys. Lett. B309 (1993) 378.

[15] K. M. Bitar et al., Phys. Rev. D46 (1992) 2169. 


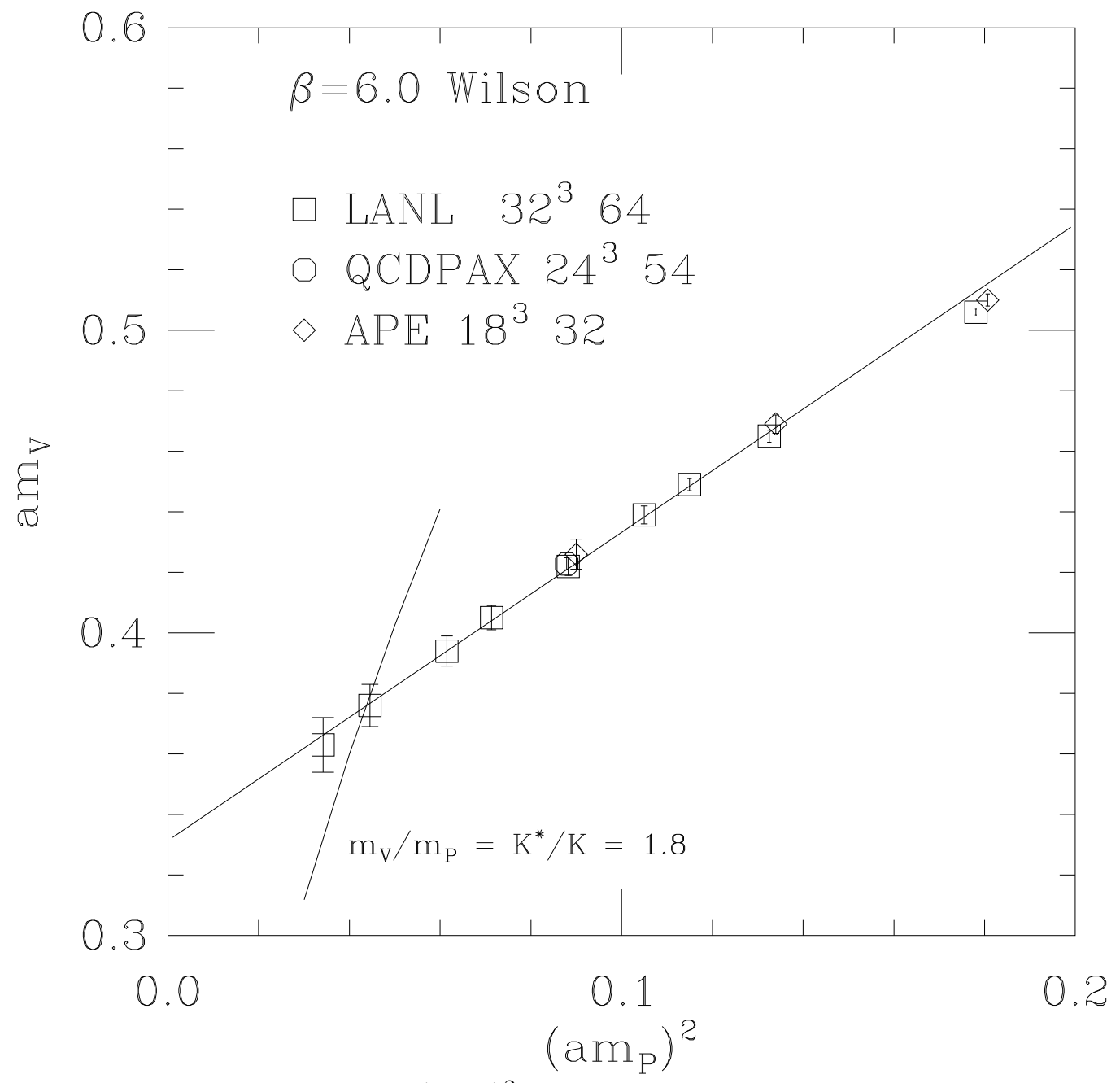

Figure 1: The value of $a m_{V}$ versus $\left(a m_{P}\right)^{2}$ in lattice units. The data are for Wilson fermions at $\beta=6.0$ from the LANL, QCDPAX and APE groups. The LANL data have pairs of unequal quark masses as well as equal. A straight line fit to the LANL data, excluding the heaviest mass point, is shown. The curve corresponds to the reference quark mass scale set by $m_{V}=1.8 m_{P}$. 


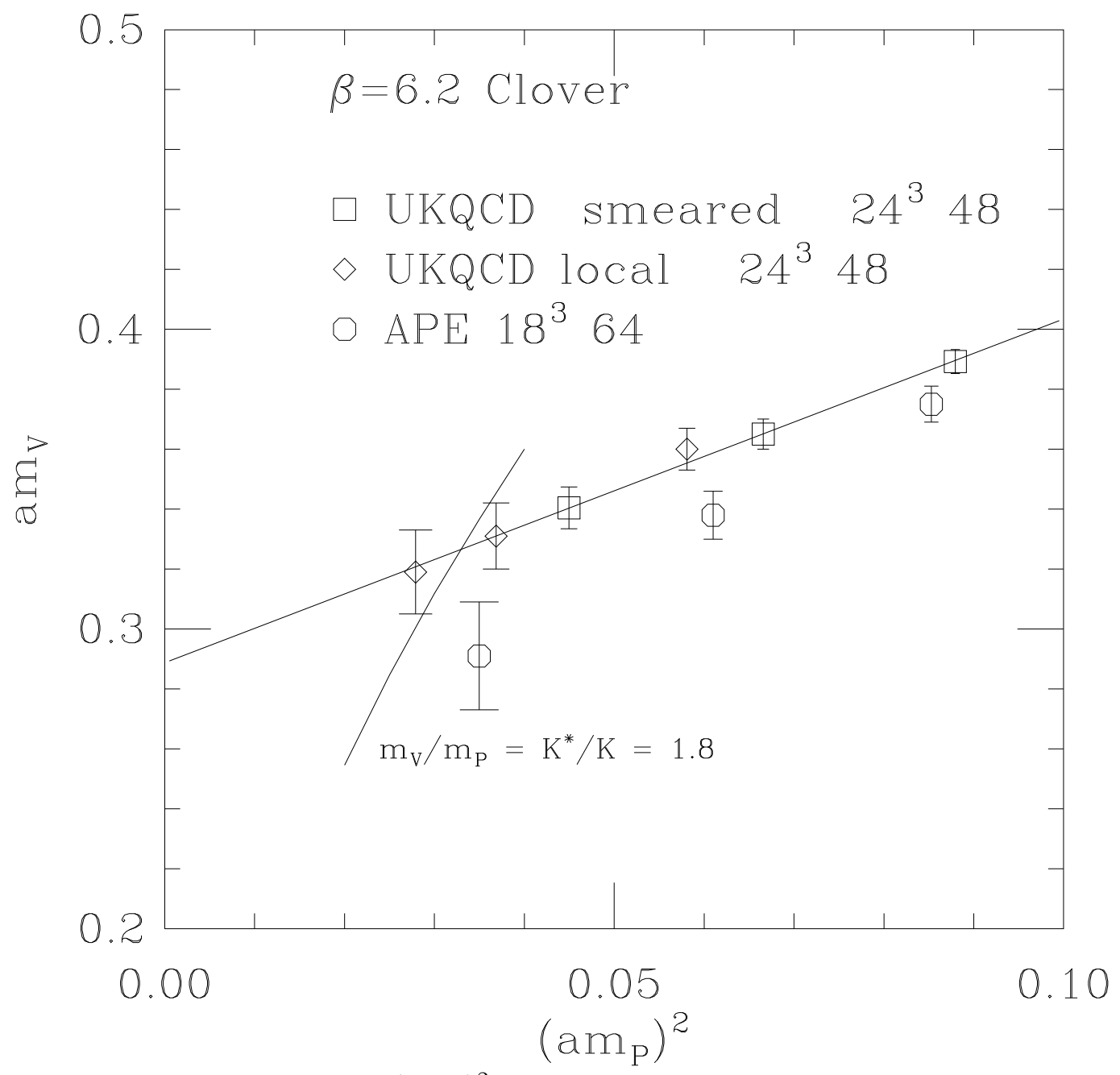

Figure 2: The value of $a m_{V}$ versus $\left(a m_{P}\right)^{2}$ in lattice units. The data are for the clover fermionic action at $\beta=6.2$ from the UKQCD and APE groups. The UKQCD data have pairs of unequal quark masses as well as equal. A straight line fit to the UKQCD data from smeared sources (squares) is shown. The curve corresponds to the reference quark mass scale set by $m_{V}=1.8 m_{P}$. 


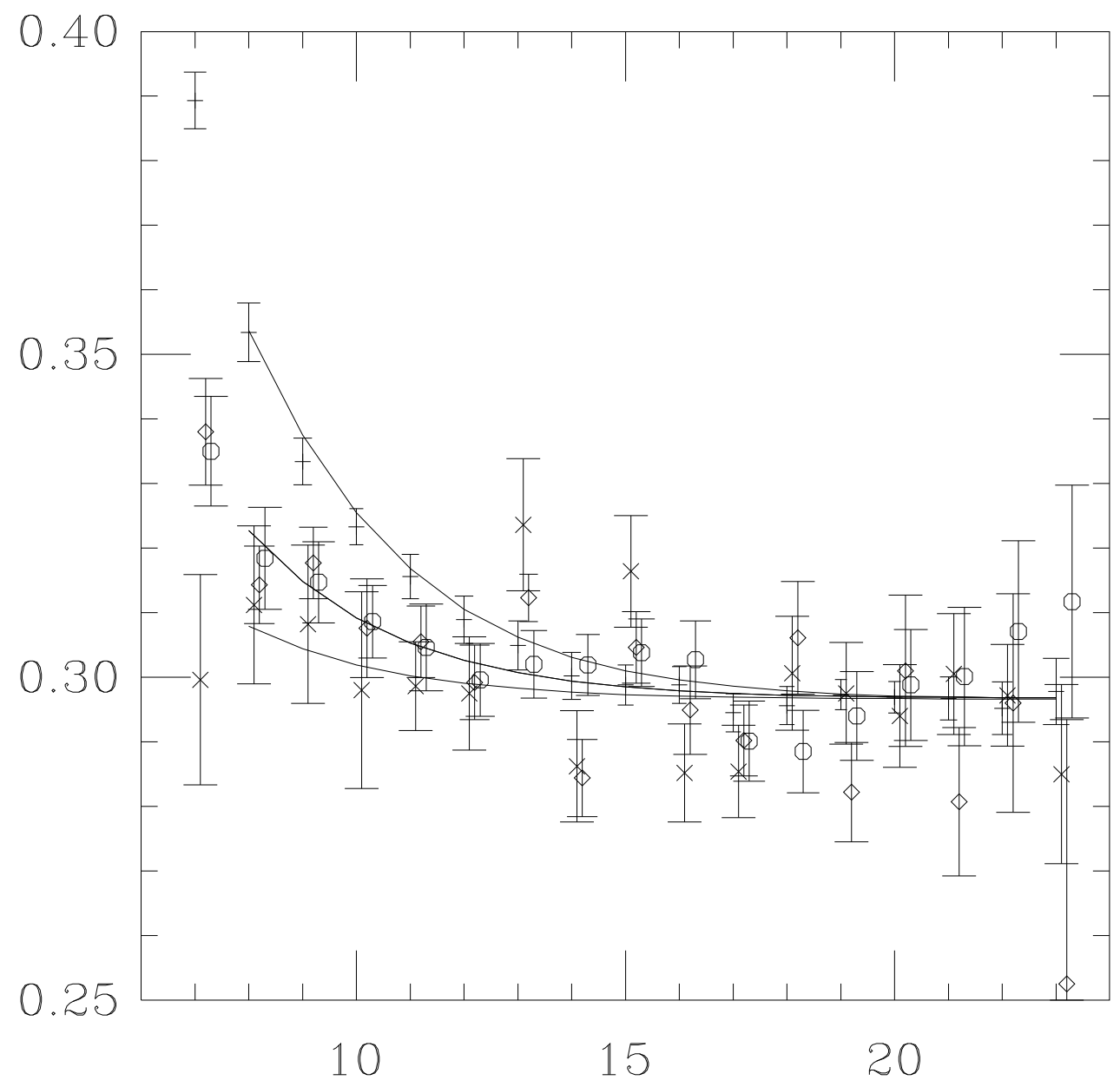

Figure 3: The effective pseudoscalar mass in lattice units from local sinks and sources (LL) versus $t$ for hopping parameter $K=0.14144$. The symbols are for spin combinations at source and sink $\mathrm{PP}(+), \mathrm{AA}(\times), \mathrm{AP}$ (octagon) and $\mathrm{PA}($ diamond). The curves represent the two-state factorising fits described in the text. 


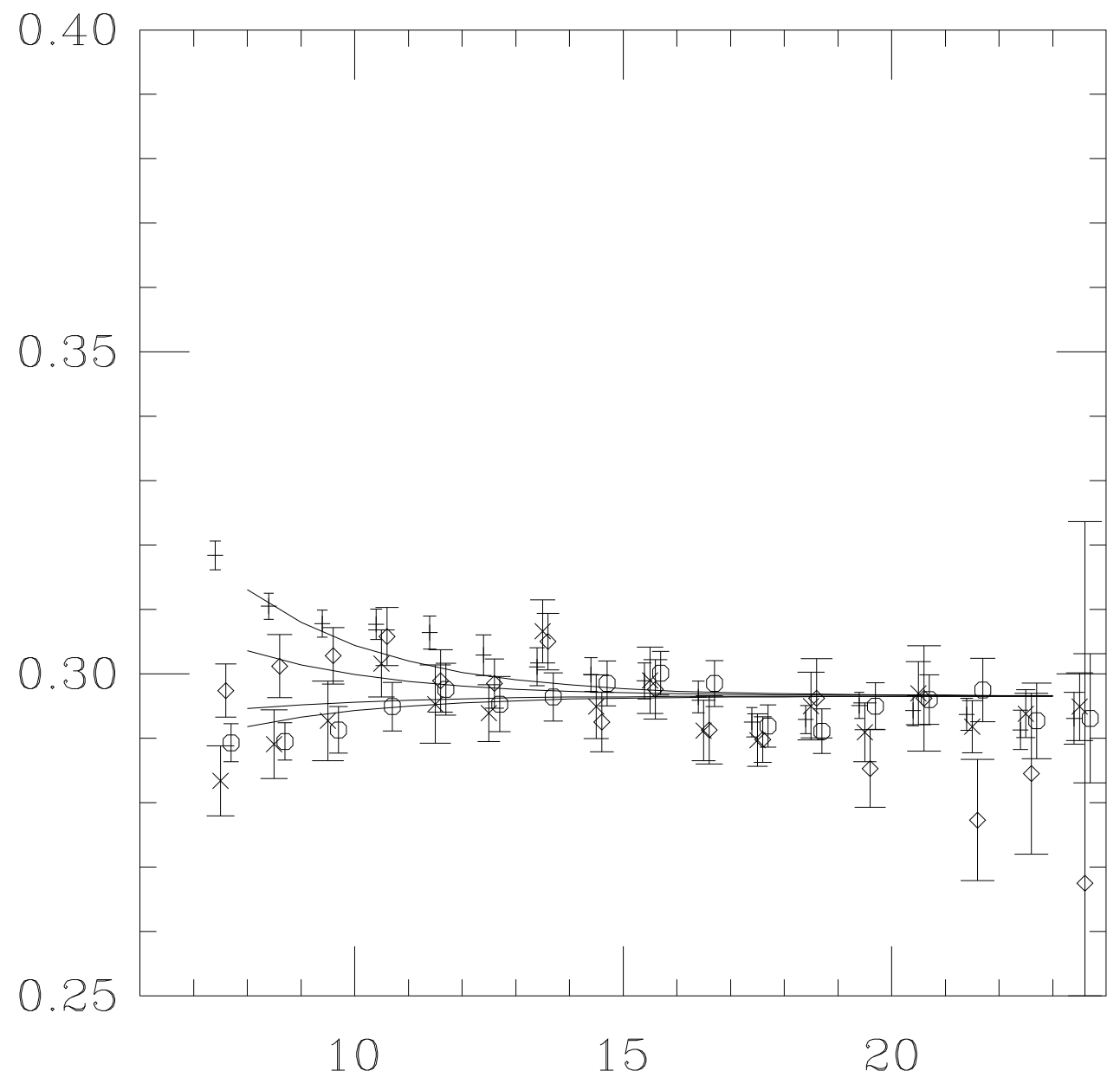

Figure 4: The effective pseudoscalar mass in lattice units from local sinks and smeared source (SL) versus $t$ for hopping parameter $K=0.14144$. The symbols are for spin combinations at source and sink $\mathrm{PP}(+), \mathrm{AA}(\times), \mathrm{AP}$ (octagon) and $\mathrm{PA}($ diamond). The curves represent the two-state factorising fits described in the text. 


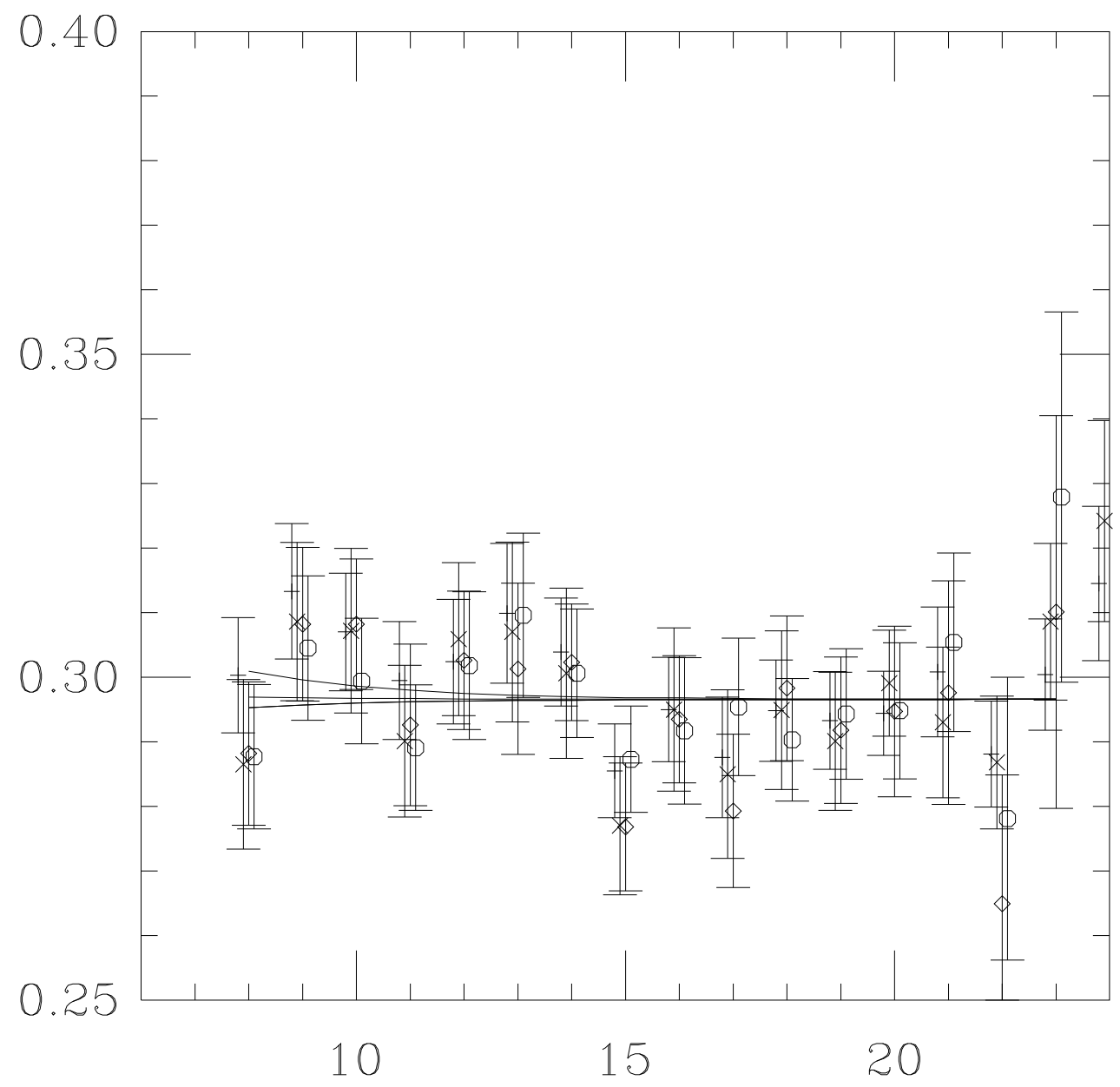

Figure 5: The effective pseudoscalar mass in lattice units from smeared sinks and sources (SS) versus $t$ for hopping parameter $K=0.14144$. The symbols are for spin combinations at source and sink $\mathrm{PP}(+), \mathrm{AA}(\times), \mathrm{AP}$ (octagon) and $\mathrm{PA}($ diamond). The curves represent the two-state factorising fits described in the text. 


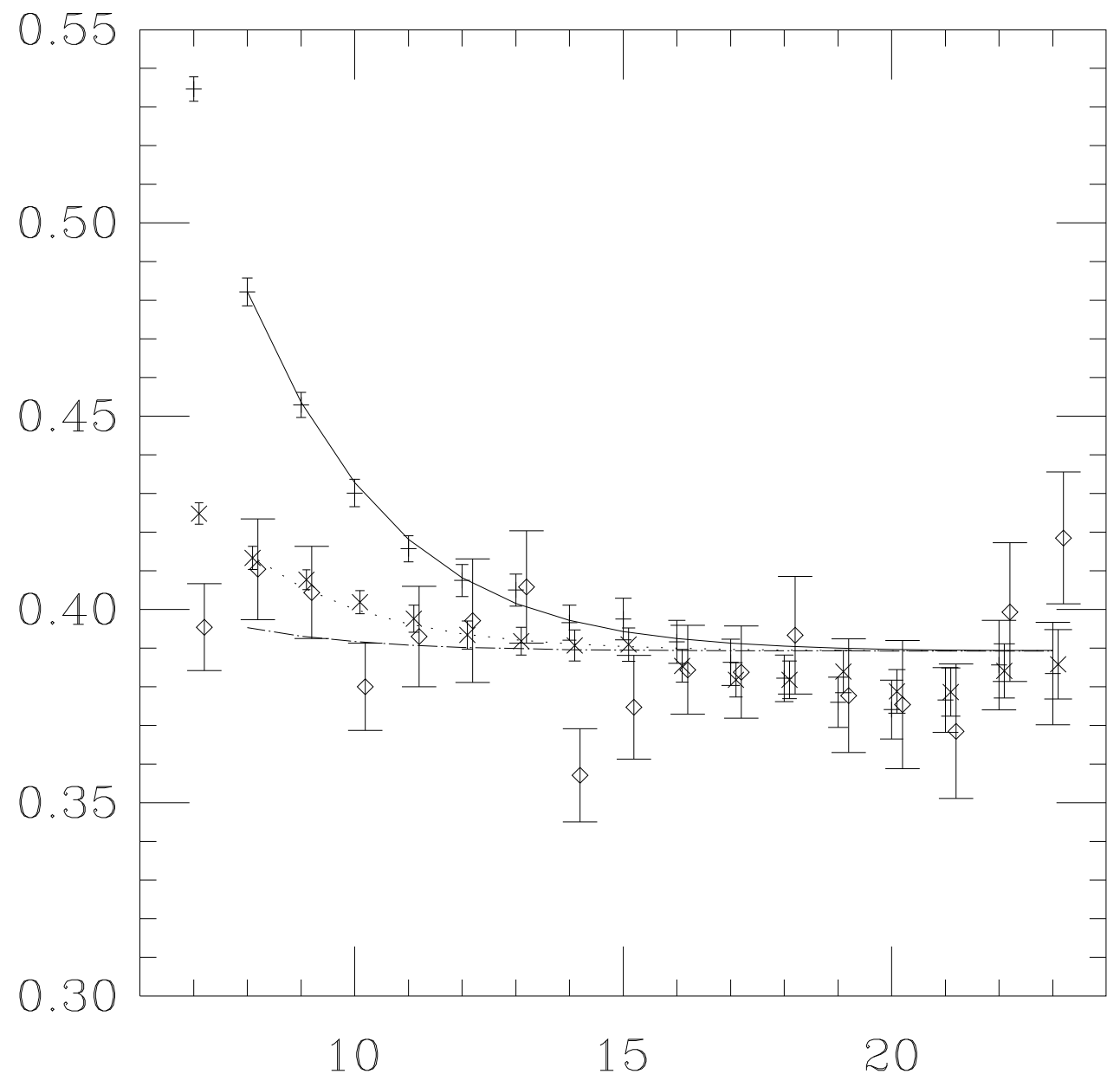

Figure 6: The effective vector mass in lattice units from local and smeared sinks and sources (SS) versus $t$ for hopping parameter $K=0.14144$. The symbols are for operators at source and sink LL $(+)$, SL $(\times)$, SS(diamond). The curves represent the two-state factorising fits described in the text. 


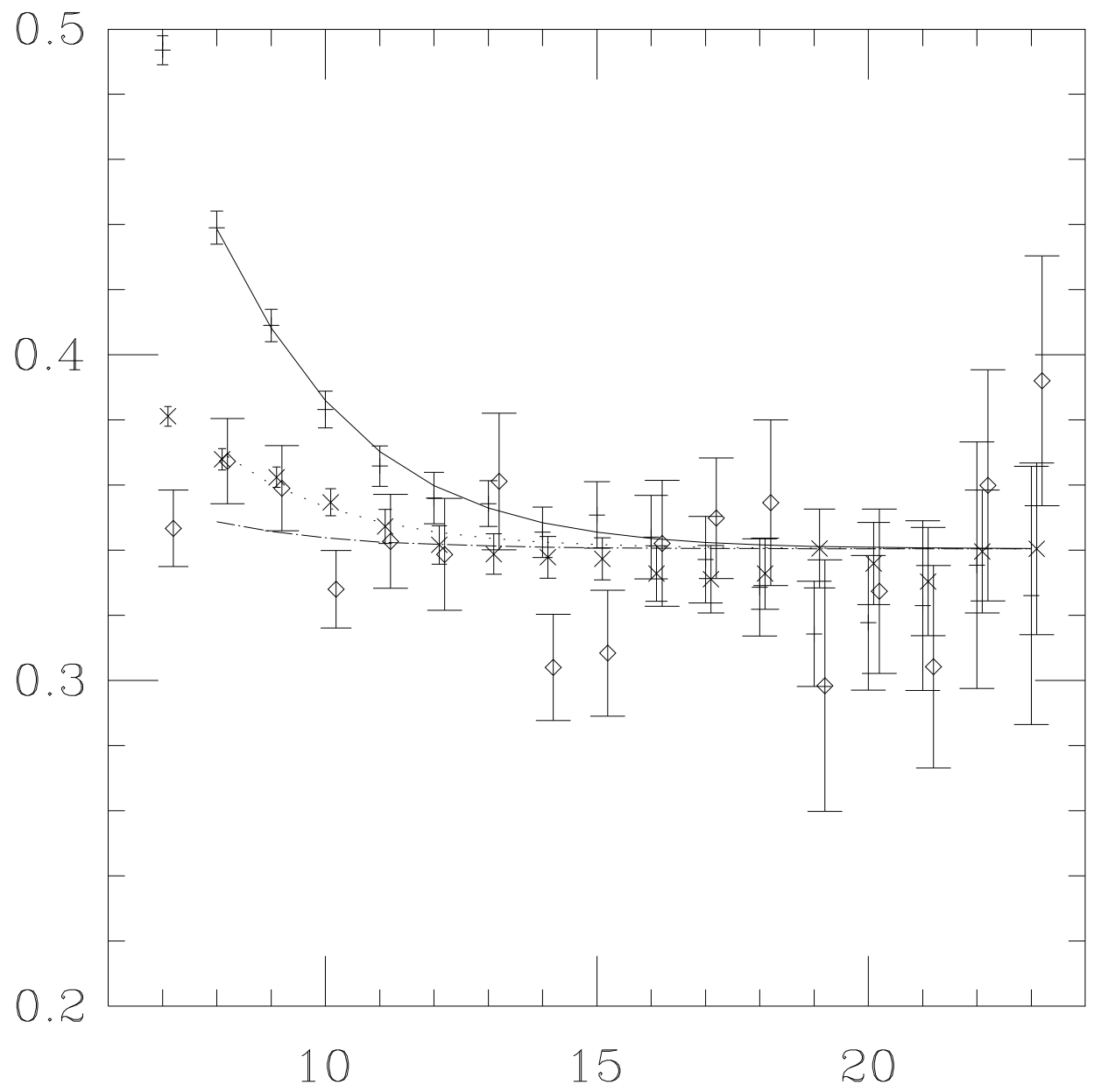

Figure 7: The effective vector mass in lattice units from local and smeared sinks and sources (SS) versus $t$ for hopping parameter $K=0.14226$. The symbols are for operators at source and sink LL $(+)$, SL $(\times)$, SS(diamond). The curves represent the two-state factorising fits described in the text. 


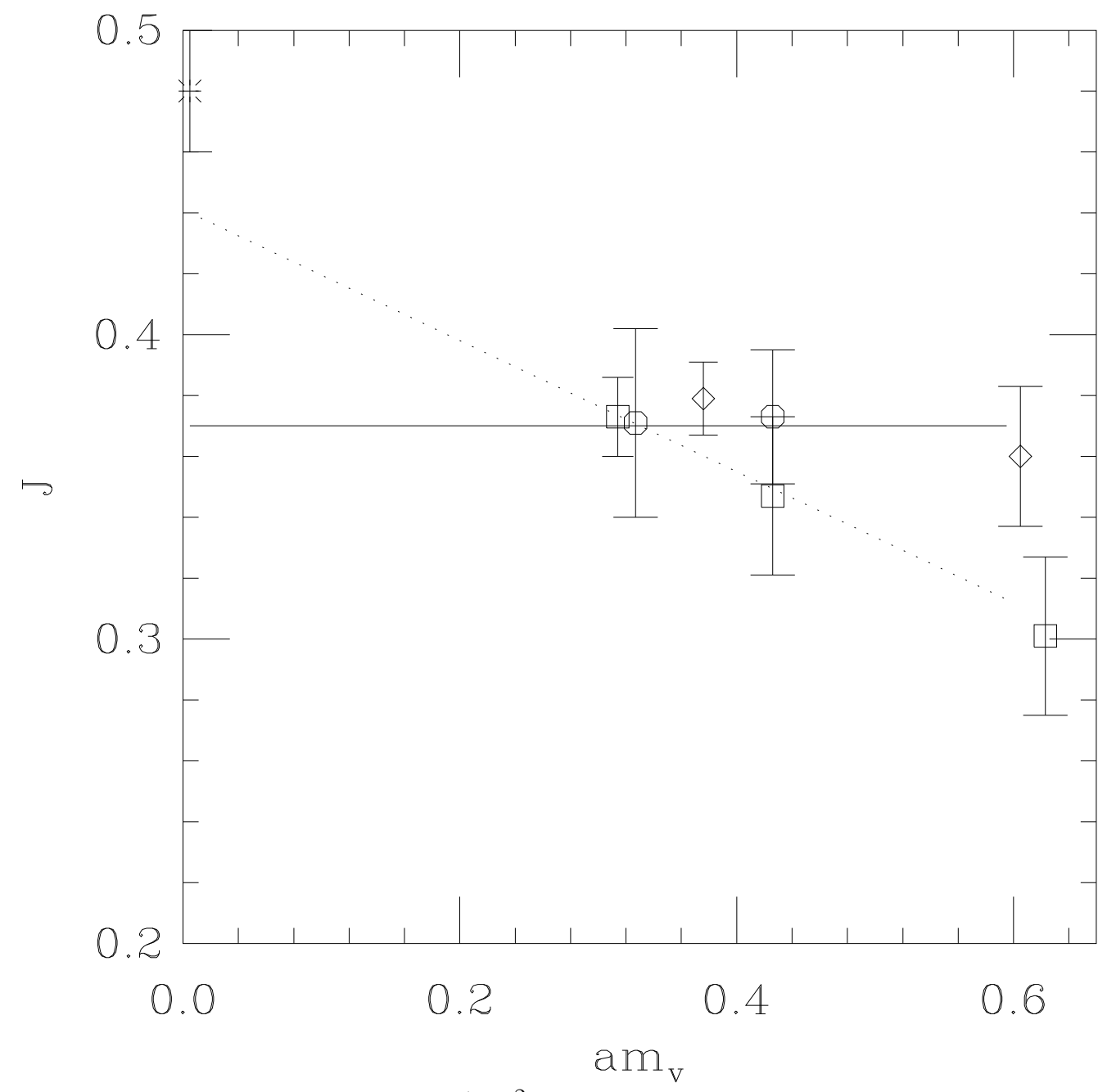

Figure 8: The value of $J=m_{v} d m_{V} / d m_{P}^{2}$ versus $m_{v}$ in lattice units. The data from clover fermions are shown by octagons from the present work (with statistical error only) and from the APE group. The data from Wilson fermions are shown by squares from the GF11 group at similar physical spatial volumes and diamonds for the larger volume results from GF11 and LANL. The cross is the experimental value. The solid and dotted lines represent different interpretations of the data, as discussed in the text. 